\title{
Associação de Lesões Anorretais em Portadoras de Infecção Genital por HPV e Neoplasia Cérvico-Uterina
}

\author{
Association Between Anorectal Lesions in Women with Genital HPV Infection \\ and Cervical-Uterine Neoplasia
}

\author{
JULIANACOTRIM AMARAL ${ }^{1}$, MARIAEUGÊNIA BARROS DE SOUZACÂMARA' ${ }^{1}$, PAULAGABRIELAMELO \\ MORAIS ${ }^{2}$, LUIZADAURAFRAGOSO DE BARROS ${ }^{3}$, MANOEL ÁLVARO DE FREITAS LINS NETO ${ }^{4}$
}
1. Doutoranda da Faculdade de Medicina da Universidade Federal de Alagoas (FAMED/UFAL), ${ }^{2}$ Residente de Cirurgia Geral do Hospital Universitário Prof. Alberto Antunes (HUPAA/UFAL), ${ }^{3}$ Professora do Departamento de Tocoginecologia e Pediatria da Universidade Federal de Alagoas, ${ }^{4}$. Professor Adjunto e Chefe do Serviço de Coloproctologia do Hospital Universitário Prof.Alberto Antunes (HUPAA/UFAL).

\begin{abstract}
AMARAL JC; CÂMARA MEBS; MORAIS PGM; BARROS LDF; LINS NETO MAF. Associação de Lesões Anorretais em Portadoras de Infecção Genital por HPV e Neoplasia Cérvico-Uterina. Rev bras Coloproct, 2009;29(2): 203-208.

RESUMO: OBJETIVOS: Diagnosticar a presença do HPV anorretal em portadoras dessa afecção no trato genital inferior, através do exame coloproctológico com anuscopia de alta resolução. MÉTODOS: Estudo observacional e transversal realizado através de um protocolo de pesquisa, no Hospital Universitário da UFAL de Fevereiro a Julho de 2008, analisando 57 mulheres com diagnóstico prévio de infecção genital por HPV submetendo-as a uma avaliação através da anuscopia de alta resolução. RESULTADOS: A idade média variou de 31,2 anos, seis pacientes eram gestantes, 59,6\% cursaram apenas $01^{\circ}$ grau, 73,7\% tinham união estável; A idade média de menarca foi 12,6 anos e a sexarca de 17 anos. Em relação ao número de parceiros sexuais, 33,3\% tiveram 2 a 3 parceiros; 68,6\% apresentaram lesão intraepitelial genital de baixo grau (LGSIL), CONCLUSÕES: 89,5\% não apresentavam lesões e 10,5\% apresentavam através da anuscopia de alta resolução(AAR) lesões sub-clínicas perianal, vulvar e vaginal. É possível e viável ao especialista incorporar o exame de anuscopia de alta resolução para diagnóstico de HPV ano-retal na forma subclínica à sua rotina, já que é de simples execução, barato e a doença acomete pacientes sem lesões visíveis ao exame proctológico.
\end{abstract}

Descritores: Papillomaviridae; Infecções por Papilomavírus; Anormalidades Urogenitais; Anuscopia ampliada; Doenças do Ânus.

\section{INTRODUÇÃO}

O papilomavírus humano (HPV) é uma das doenças sexualmente transmissíveis (DSTs) de maior incidência e prevalência no mundo, sendo a infecção por este vírus o principal fator de risco para o câncer cervical (CC). É o terceiro câncer de maior prevalência entre as mulheres. Cerca de $80 \%$ dos novos casos ocorrem nos países em desenvolvimento, e no Brasil, tal doença fica em segundo lugar, perdendo apenas para o câncer da mama. ${ }^{1,2}$
A infecção pelo HPV é altamente prevalente, sendo detectada em aproximadamente $10 \%$ a $20 \%$ da população sexualmente ativa entre 15 e 49 anos de idade. ${ }^{2,3}$

Atualmente, são conhecidos mais de 100 subtipos de HPV, destes, 30 possuem tropismo pelo trato anogenital, e são divididos em dois grupos de acordo com o seu potencial de oncogenicidade. Os de baixo risco (HPV tipo 6, 11, 42, 43 e 44), incluindo os condilomas genitais, dos quais os tipos 6 e 11 são os mais prevalentes, assim como os de alto risco

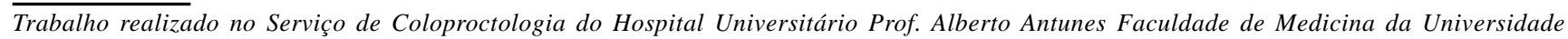
Federal de Alagoas. Maceió, Alagoas - Brasil. 
oncogênico (HPV tipo 16,18,31,33,35,39,45,52,56). Quando associados a outros co-fatores, têm relação com o desenvolvimento das neoplasias intra-epiteliais e do câncer invasor do colo uterino, vulva, vagina e região anal ${ }^{1,4,5}$. Os fatores de risco clássicos para essas neoplasias são basicamente os mesmos relacionados à infecção pelo HPV, dentre eles, destacam-se: início precoce das relações sexuais, vários parceiros sexuais ao longo da vida, paridade elevada (partos não cirúrgicos), ser jovem, tabagismo, baixo nível socioeconômico, uso de anticoncepcional oral prolongado, deficiências nutricionais, infecção por HIV (vírus da imunodeficiência humana) e outras infecções genitais causadas por agentes sexualmente transmissíveis. $^{3}$

Diferentemente dos outros cânceres humanos, o câncer cervical é, em princípio, doença evitável, já que apresenta evolução lenta, com longo período desde o desenvolvimento das lesões precursoras ao aparecimento do câncer. Apesar da eficácia dos programas de controle de câncer cérvico-uterino, pela realização de colpocitologia oncótica, o carcinoma cervical mantém-se como doença de alta prevalência, incidência e mortalidade. ${ }^{6}$

Os papilomavírus humanos infectam a pele e a membrana das mucosas, podendo ser transmitidos por contato cutâneo, sexual ou perinatal, a depender do tipo de HPV e das lesões clínicas associadas a ele, e consequentemente, induzir a formação de tumores epiteliais benignos e malignos. Além do trato genitourinário, esta infecção apresenta-se sob formas cutânea, oftálmica, respiratória superior e digestória. Dentre estas formas, o ânus é o sítio extragenital mais comum para cepas genitais da infecção pelo HPV. ${ }^{7}$

Estudos preliminares demonstraram que mulheres com infecção por HPV no colo uterino apresentam risco duas vezes maior para a presença de infecção por HPV anal concomitante. Então, mulheres jovens praticantes de sexo anal ou com história positiva para neoplasia intraepitelial cervical (NIC) apresentaram risco maior para citologias anais anormais, portanto, observou-se que mulheres com lesões de alto grau cervicais, vaginais ou vulvares, podem beneficiar-se do rastreamento anal. ${ }^{8}$

A maioria das infecções pelo HPV não tem qualquer consequência clínica, mas cerca de $10 \%$ dos pacientes desenvolverá verrugas, papilomas ou displasias, porém, na zona de transição do canal anal é considerada doença pré-cancerígena, com a possibili- dade de progressão de carcinoma "in situ" para invasor. $^{5}$

O diagnóstico e tratamento são ainda precários, levando a traumas psico-sociais e físicos, com repercussões na produtividade, sexualidade, equilíbrio sócio-familiar e outros índices de saúde, bem como o aumento da incidência de câncer. ${ }^{4}$

Com o aumento da incidência desta infecção na população sexualmente ativa e de suas repercussões gerais à paciente, foi proposto um estudo transversal, a fim de conhecer melhor o comportamento desta doença, e contribuir para ações voltadas à apresentação e seu controle. Com este trabalho, objetivamos diagnosticar a infecção pelo HPV anorretal em portadoras dessa infecção no trato genital inferior, por meio de exame coloproctológico com anuscopia de alta resolução, para prevenção do câncer anogenital.

\section{CASUÍSTICA E MÉTODOS}

Trata-se de um estudo observacional e transversal onde foram estudadas 57 mulheres sexualmente ativas, com critério de inclusão de diagnóstico clínico, citológico, colposcópico e histopatológico prévio de infecção genital por HPV, atendidas no Serviço de Ambulatório de Patologia do Trato Genital Inferior e Colposcopia (PTGIC), do Serviço de Ginecologia do Hospital Universitário Professor Alberto Antunes (HUPAA - UFAL), no período de Fevereiro a Julho de 2008. Este trabalho foi submetido e aprovado pelo Conselho de Ética da Universidade.

Os dados foram obtidos através do preenchimento de um protocolo de pesquisa, com a assinatura do termo de consentimento livre e esclarecido. As mulheres foram posteriormente encaminhadas ao ambulatório de coloproctologia do HUPAA - UFAL, e submetidas a anuscopia de alta resolução com um aparelho de colposcopio, o mesmo utilizado para os exames ginecológicos.Esta alternativa amplia em 10 vezes as possíveis lesões que não são visíveis ao exame proctológico convencional..O exame consiste na aplicação na pele e mucosa anorretal de ácido acético a 5\% por dois minutos, retira-se o excesso do ácido e a seguir aplica-se azul de toluidina, após realizada esta etapa investigamos as lesões com colposcópio. Os casos que apresentaram lesões perianais suspeitas de HPV foram biopsiados e seu material enviado para o Serviço de Histopatologia do HUPAA - UFAL. As mulheres diagnosticadas com lesão anorretal foram 
devidamente tratadas. $\mathrm{O}$ banco de dados foi analisado através do programa SPSS (Statistical Package for Social Sciences) for windows, versão 12.0.

\section{RESULTADOS}

Entre as 57 pacientes estudadas, com média de idade de 31,26 anos (18-61 anos), seis (10,5\%) eram gestantes. Duas $(3,5 \%)$ mulheres nunca frequentaram a escola, $34(59,6 \%)$ frequentaram o primeiro grau e $21(36,9 \%)$ cursaram o segundo grau. Quarenta e duas pacientes $(73,7 \%)$ tinham situação marital tipo união estável, 14 $(24,5 \%)$ eram solteiras, e apenas uma $(1,8 \%)$ divorciada. Quanto ao tabagismo $12(21,1 \%)$ mulheres afirmaram o seu consumo, e $45(78,9 \%)$ negaram. (Tabelas 1 e 2)

A faixa etária média de menarca foi 12,67 anos (10-16anos), enquanto a idade de início das relações sexuais foi 17,09 anos (11-30anos). Em relação ao número de parceiros sexuais, $16(28,1 \%)$ mulheres tiveram apenas um parceiro, $19(33,3 \%)$ de dois a três

Tabela 1 - Distribuição das pacientes de acordo com a escolaridade.

\begin{tabular}{lrr}
\hline Escolaridade & N & \multicolumn{1}{c}{$\%$} \\
\hline Analfabeta & 2 & 3,5 \\
$1^{\circ}$ Grau & 34 & 59,6 \\
$2^{\circ}$ Grau & 21 & 36,9 \\
Total & 57 & 100,0 \\
\hline
\end{tabular}

Tabela 2 - Distribuição das pacientes segundo o estado civil.

\begin{tabular}{lrc}
\hline Estado Civil & N & \% \\
\hline União estável & 42 & 73,7 \\
Divorciada & 1 & 1,8 \\
Solteira & 14 & 24,5 \\
Total & 57 & 100,0 \\
\hline
\end{tabular}

parceiros, $14(24,6 \%)$ de quatro a cinco, e oito (14\%), seis ou mais parceiros. Vinte $(35,1 \%)$ fizeram uso prolongado de contraceptivos orais, enquanto $37(64,9 \%)$ nunca utilizaram este meio. Apenas $19(33,3 \%)$ pacientes afirmaram o uso de preservativo e $38(66,7 \%)$ não relataram o uso regular deste método de barreira. Dez $(17,5 \%)$ pacientes confirmaram a prática do coito anal e $47(82,5 \%)$ negaram. (Tabelas 3, 4 e 5).

Quarenta e sete $(82,5 \%)$ negaram o passado de outras doenças sexualmente transmissíveis, além do HPV, uma paciente $(1,8 \%)$ afirmou ser portadora de hepatite $\mathrm{B}$, uma $(1,8 \%)$ de Herpes genital, uma $(1,8 \%)$ de HIV, duas $(3,5 \%)$ sífilis e cinco $(8,8 \%)$ em forma de corrimento vaginal. Quinze pacientes $(26,3 \%)$ queixaram-se de prurido anal, enquanto $42(73,7 \%)$ não referiram tal sintoma. (Tabela 6)

Tabela 4 - Distribuição das pacientes de acordo com o número de parceiros.

\begin{tabular}{lrc}
\hline Parceiros & N & \% \\
\hline 1 & 16 & 28,1 \\
2 A 3 & 19 & 33,3 \\
4 A 5 & 14 & 24,6 \\
6 ou mais & 8 & 14,0 \\
Total & 57 & 100,0 \\
\hline
\end{tabular}

Tabela 5 - Relação entre o Coito Anal e a presença de condilomatose perianal.

\begin{tabular}{lccc}
\hline Coito anal & \multicolumn{2}{c}{ Diagnóstico Proctológico } & Total \\
\cline { 2 - 3 } & Negativo & Positivo & \\
\hline Não & 43 & 4 & 47 \\
Sim & 8 & 2 & 10 \\
T o t a l & 51 & 6 & 57 \\
\hline
\end{tabular}

Para verificar se existe diferença entre coito anal e diagnóstico proctológico, foi utilizado o teste exato de Fisher, obtendo-se um valor de $p=0,281$. Este resultado indica que não existe diferença significativa entre coito anal e diagnóstico proctológico $(p>0,05)$.

Tabela 3 - Distribuição de acordo com a faixa etária e idades de menarca e sexarca.

\begin{tabular}{lcccc}
\hline & Mínimo & Máximo & Média & Desvio Padrão \\
\hline Idade & 18 & 61 & 31,26 & 9,86 \\
Menarca & 10 & 16 & 12,67 & 1,37 \\
Sexarca & 11 & 30 & 17,09 & 3,51 \\
\hline
\end{tabular}


Tabela 6 - Relação de Prurido anal com a presença de condiloma anal.

\begin{tabular}{llccc}
\hline & & \multicolumn{2}{c}{ Condiloma Anal } & Total \\
\cline { 3 - 4 } & & Negativo & Positivo & \\
\hline Prurido Anal & Não & 42 & & 42 \\
\multirow{2}{*}{ Total } & 9 & 6 & 15 \\
& Sim & 51 & 6 & 57 \\
\hline
\end{tabular}

Em nossa casuística, dezoito (31,6\%) apresentavam lesão intraepitelial genital de alto grau (HGSIL) e $39(68,6 \%)$ lesão intraepitelial genital de baixo grau (LGSIL), seis mulheres (LG-ASIL), $10,5 \%$, apresentavam HPV anorretal através da AAR e $51(89,5 \%)$ foram negativas para infecção anal. (Tabelas 7,8 e 9).

\section{DISCUSSÃO}

O papilomavírus humano (HPV) é a doença infecciosa do trato genital feminino, de transmissão

Tabela 7 - Distribuição de lesões genitais encontradas no estudo.

\begin{tabular}{lcc}
\hline & Frequência & Percentual \\
\hline Baixo grau (LGSIL) & 39 & 68,4 \\
Alto grau (HGSIL) & 14 & 24,6 \\
Carcinoma Invasor & 4 & 7,0 \\
Total & 57 & 100,0 \\
\hline
\end{tabular}

Tabela 8 - Frequência de lesões anais.

\begin{tabular}{lcc}
\hline & Frequência & Percentual \\
\hline Negativo & 51 & 89,5 \\
Positivo & 6 & 10,5 \\
Total & 57 & 100,0 \\
\hline
\end{tabular}

sexual, mais prevalente entre as mulheres de vida sexual ativa, no período reprodutivo, compondo mais de 100 subtipos diferentes, dos quais 25 têm a capacidade de infectar a região anogenital, e uma porção expressiva destes está vinculada ao potencial oncogênico. $5,9,10$

Entre as 57 pacientes estudadas, a média de idade foi de 31,26 anos, o que está em acordo com a literatura sobre o assunto, e corresponde à faixa etária de maior prevalência da infecção genital pelo HPV. Seis $(10,5 \%)$ destas pacientes eram gestantes, condição que exerce uma imunossupressão transitória, responsável pela recrudescência do condiloma ${ }^{10,11}$. Grande parte das participantes da pesquisa cursou apenas o ensino fundamental, refletindo o grau de escolaridade e o nível de renda como principais indicadores das condições socioeconômicas da doença estudada. De acordo com o presente estudo, no que se refere à situação conjugal, depreende-se a concordância com a literatura que a frequência mais elevada ocorre em mulheres casadas, principalmente na progressão para câncer de colo.

Quanto ao tabagismo, apenas uma menor parcela do grupo, relatou o seu consumo, mostrando que apesar de alguns autores relacionarem este fator de risco ao desenvolvimento de NIC e câncer cervical, não houve significância estatística em nosso estudo.

Apesar de existirem controvérsias quanto aos fatores relacionados às formas de contágio pelo HPV,

Tabela 9 - Associação de lesões anais nas pacientes com lesões genitais por HPV.

\begin{tabular}{|c|c|c|c|}
\hline \multirow[t]{2}{*}{ Diagnóstico ginecológico } & \multicolumn{2}{|c|}{ Condiloma } & \multirow[t]{2}{*}{ Total } \\
\hline & Negativo & Positivo & \\
\hline Baixo grau (LGSIL) & 33 & 6 & 39 \\
\hline Alto grau (HGSIL) & 14 & & 14 \\
\hline Carcinoma Invasor & 4 & & 4 \\
\hline Total & 51 & 6 & 57 \\
\hline
\end{tabular}


Associação de Lesões Anorretais em Portadoras de Infecç̃̃o Genital por HPV e Neoplasia Cérvico-Uterina Juliana Cotrim Amaral e Cols. sua persistência e o aparecimento de lesões histológicas, existem evidências muito fortes demonstrando que o HPV é o principal agente etiológico do câncer de colo de útero e câncer anorretal, assim como de suas formas pré-invasivas. ${ }^{1,6,12}$

Vários trabalhos correlacionam a hipótese etiológica entre o HPV e o câncer cervical. Entre os fatores de risco mais conhecidos estão a atividade sexual, tais como: relacionamento sexual precoce, idade jovem na primeira gravidez, número de parceiros sexuais, alta paridade e uso prolongado de contraceptivos hormonais orais, porém isto deve-se não só aos efeitos da pílula, mas provavelmente também ao comportamento sexual deste grupo de mulheres. ${ }^{13,12}$ Todos esses fatores foram identificados em concordância com o contexto estudado.

Dez $(17,5 \%)$ pacientes confirmaram a prática do coito anal, duas apresentaram lesão anal, indicando que, embora este fator comportamental esteja implicado na gênese das lesões escamosas intraepiteliais anogenitais condizentes com a literatura, não foi possível correlacioná-las devido a pequena amostra em questão.

Devido à inconsistência dos dados obtidos através do protocolo de pesquisa sobre o passado de doenças sexualmente transmissíveis (DSTs), não houve neste estudo, correlação direta desse fator associado a lesões precussoras de câncer cervical. Conforme descrito na literatura, o processo inflamatório crônico no tecido acometido, sobre um epitélio geneticamente suscetível, pode ser fator de promoção da carcinogênese. ${ }^{14,15}$

Todos esses fatores somados as dificuldades de acesso aos meios de diagnósticos e tratamento precoce, confere a esta população uma alta incidência de

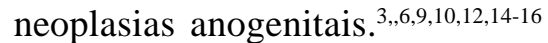

Queixa bastante frequente entre as pacientes estudadas foi o prurido anal, referido por 15 mulheres
(26,3\%). Sabemos que a forma clínica do HPV anal ou condiloma anal, caracterizada pelas verrugas, é uma causa de prurido, e que as manifestações de forma latente do HPV anal ainda são desconhecidas por não haver métodos práticos que localizem as áreas contaminadas pelo vírus. No entanto, a forma subclínica do HPV anal, apesar de imperceptível macroscopicamente, pode ser diagnosticada pela anuscopia de alta resolução. $\mathrm{O}$ fato é que este exame não é de uso comum na rotina do especialista, levando esta doença a ser subestimada como diagnóstico etiológico diferencial de prurido anal.

A infecção pelo HPV e a subsequente integração do DNA viral são necessárias, mas não suficientes para causar progressão para câncer, embora os HPV de alto risco sejam causa necessária para o câncer cervical, eventos genéticos adicionais são indispensáveis para tranformação maligna na maioria dos carcinomas anais e de outros sítios. Deste modo, com esta pesquisa tentou-se contribuir para a elucidação de grupos de mulheres com risco de infecção por HPV, que mereçam avaliação do canal anal, através da anuscopia de alta resolução, para a prevenção e propedêutica do câncer anogenital.

\section{CONCLUSÃO}

Em nossa casuística das pacientes com diagnóstico ginecológico de infecção por HPV, em seis pacientes, $(10,5 \%)$ foi constatada presença de lesões condilomatosas sub-clínicas através da anuscopia de alta resolução.

É possível e viável ao especialista incorporar o exame de anuscopia de Alta Resolução para diagnóstico de HPV ano-retal na forma subclínica à sua rotina, já que é de simples execução, barato e a doença acomete pacientes sem lesões visíveis ao exame proctológico.

\footnotetext{
ABSTRACT: AIM: To diagnose the presence of anorectal HPV in women with lower genital tract lesions, through rectal examination with High Resolution Anuscopy. METHODS: Observational, transversal study conducted by a research protocol in the Hospital Universitário Prof. Alberto Antunes (HUPAA/UFAL) from February to July 2008, examining 57 women with a previous diagnosis of genital HPV infection by subjecting them to an assessment by High Resolution Anuscopy. RESULTS: The mean age ranged from 31.2 years, six patients were pregnant women, $\mathbf{5 9 . 6 \%}$ had finished elementary school, $\mathbf{7 3 . 7 \%}$ were in a stable union with a partner, the average age of menarche was 12.6 years and 17 years for the first intercourse. Regarding the number of sexual partners, 33.3\% had 2 to 3 partners, $68.6 \%$ had low grade squamous intraepithelial lesion (LGSIL), CONCLUSIONS: 89,5\% had no lesions and $\mathbf{1 0 , 5} \%$ had subclinical perianal, vulvar and vaginal lesions. It is possible and feasible to the professionals incorporate the High Resolution Anuscopy for diagnosis of HPV in anorectal subclinical lesions to their routine, it is simple,inexpensive and mainly because the disease affects patients with no visible lesions to the anorectal examination.
}

Key words: Papillomaviridae; Papillomavirus Infections ; Urogenital Abnormalities; Rectal diseases; Anus diseases. 


\section{REFERÊNCIAS}

1. Albring L, Brentano JE, Vargas, VRA. O câncer do colo do útero, o Papilomavírus Humano (HPV) e seus fatores de risco e as mulheres indígenas Guarani: estudo de revisão. RBAC, 2006; 38(2): 87-90.

2. Bezerra SJS, Gonçalves PC, Franco, ES; et al. Perfil de mulheres portadoras de lesões cervicais por HPV quanto aos fatores de risco para câncer de colo de útero. DST - J brás. Doenças Sex Transm. 2005,17(2): 143-148.

3. Pinto AP, Tulio S, Cruz OR. Co-fatores do HPV na oncogênese cervical., Rev. Assoc. Med. Bras. 2002, 48 (1).

4. Magi JC, Brito SEM, Grecco ETO et al. Prevalência de papilomavirus humano (HPV) anal, genital e oral, em ambulatório geral de coloproctologia., Rev bras. Coloproct., 2006,26 (3): 233-238.

5. Magi JC, Magi DAS, Reche LMC et al. Anuscopia com exacerbação para diagnóstico de Papilomavirus humano ano-retal na forma subclínica. Rev bras Coloproct, 2002; 22(3): 178183.

6. Derchain SFM, Longatto Filho A, Syrjanen, KJ. Neoplasia intra-epitelial cervical: diagnóstico e tratamento. Rio de Janeiro, Rev. Bras. Ginecol. Obstet, 2005,27 (7).

7. Wieland U, Pfister H. Papilomavírus em patologia humana: epidemiologia, patogênese e papel oncogênico. In: GROSS, G.E. \& BARRASO, R. Infecção por Papilomavírus Humano: Atlas Clínico de HPV. Porto Alegre: Artmed, 1999. cap.1, p.118

8. Jacyntho C, Giraldo P. A Importância do exame citoanuscópico sob visão ampliada para o diagnóstico das neoplasias intra-epiteliais anais em pacientes com neoplasias intra-epiteliais genitais. Rio de Janeiro Rev. Bras. Ginecol. Obstet. 2005, 27(1).
9. Nadal SR, Manzione CR. Papilomavirus Humano e o Câncer Anal. Rev bras Coloproct, 2006; 26(2): 204-207.

10. Marana HRC, Duarte G, Quintana MS. Fatores de risco para recidiva após tratamento de lesões provocadas pelo HPV no trato genital feminino. Rio de Janeiro, Rev. Bras. Ginecol. Obstet, 1999; 21(4).

11. Franco ELF. Epidemiologia das Verrugas Anogenitais e do Câncer. In: LORINCZ, A.T. \& REID, R. Papilomavírus Humano. Rio de Janeiro: Interlivros, 1997. (2):19 - 41.

12. Pinotti JA, Fonseca AM.; Begnoli,VR. Tratado de Ginecologia: Condutas e Rotinas da Disciplina de Ginecologia da Faculdade de Medicina da Universidade de São Paulo.São Paulo: Revinter, 2005.

13. Aleixo Neto A. Aspectos epidemiológicos do câncer cervical. São Paulo, Rev. Saúde Pública, 1991, 25(4).

14. Nadal SR, Manzione CR. Os Agentes Sexualmente Transmissíveis e o Câncer Anorretal. Rev bras Coloproct, 2004; 24 (3): 274-277.

15. Figueiredo EMA.Ginecologia Oncológica. Rio de Janeiro: Revinter, 2004.

16. Nadal SR, Calore EE, Manzione C R et al. Incidência de Neoplasias Intraepiteliais Anais em Doentes HIV-Positivos Portadores de Condilomas Acuminados, Comparando Período Anterior e Posterior aos Inibidores da Protease. Rev bras Coloproct, 2005, 25(3): 217-222.

\section{Endereço para correspondência:}

MANOEL ÁLVARO DE FREITAS LINS NETO

Rua Profa Higia Vasconcelos 18/503

Ponta Verde-Maceio - AL

E-mail: mlinsneto@gmail.com 